\title{
Eficacia de la higiene de manos con un preparado de base alcohólica vs lavado de manos con agua y jabón
}

\author{
Effectiveness of alcohol-based handrub vs \\ handwashing with soap and water
}

\author{
Ángela Liliana Londoño, Marta lucia Murillas • Armenia (Colombia)
}

\section{Resumen}

Introduccion: la eficacia de los preparados de base alcohólica para manos con etanol al $80 \%$ o alcohol isopropílico al 75\% como alternativa al lavado de manos con jabón normal o antimicrobiano constituyen el objeto del Reto Mundial en pro de la Seguridad del Paciente: "Una atención limpia es una atención más segura".

Objetivo: medir eficacia para eliminación de gérmenes del preparado de base alcohólica para manos propuesto por la Organización Mundial de la Salud.

Materiales y metodos: se incluyeron 103 trabajadores sanitarios, $17.5 \%$ médicos y $82.5 \%$ enfermeras que laboraban en un hospital. Se tomaron 332 muestras antes y después de cada técnica.

Resultados: se identificaron $28.3 \%$ muestras con unidades formadoras de colonias previo a lavado de manos y $15.3 \%$ en la posterior $(p=0.234)$; con preparado de base alcohólica para manos en la muestra previa $25.6 \%$ y después de la higiene $2.4 \%(p=0.000)$. Se identificaron Staphylococcus coagulasa negativo en el $25.9 \%$ y Gram negativos en $2.1 \%$ de las muestras, en 4 casos se aisló Enterobacter sp. La receta de base alcohólica para manos fue eficaz antes y después de la higiene con preparado de base alcohólica (RR 0.10 IC 95\% 0.02-0.39), al compararla con el lavado con agua y jabón (RR 0.12 IC 95\% 0.03-0.5). La técnica de lavado de manos no fue eficaz (RR 0.71 IC $95 \%$ 0.43- 1.18).

Conclusion: el preparado de base alcohólica para manos hecho con la receta sugerida por la Organización Mundial de la Salud es más eficaz en la eliminación de gérmenes de las manos que el lavado de manos con jabón. (Acta Med Colomb 2011; 36: 181-186)

Palabras clave: lavado de manos, infección hospitalaria, infecciones nosocomiales, transmisión de enfermedad infecciosa de profesional a paciente, control de infecciones, control de enfermedades transmisibles, atención a la salud.

\footnotetext{
Abstract

Introduction: The effectiveness of alcohol-based handrub containing $80 \%$ ethanol or isopropyl alcohol $75 \%$, as an alternative to handwashing with plain soap and water is the objective of global challenge for the safety of the patient, "Clean Care is Safer Care".

Objective: To measure effectiveness on removing germs of Alcohol-based handrub proposed by the World Health Organization.

Materials and methods: We included 103 health workers, $17.5 \%$ physicians and $82.5 \%$ nurses who worked in a hospital. 332 samples were taken before and after each technique

Results: $28.3 \%$ samples were identified coloni forming units before handwashing and $15.3 \%$ in the posterior $(\mathrm{p}=0.234)$, with alcohol-based handrub in the sample previous $25.6 \%$ and after the hygiene $2.4 \%$ ( $\mathrm{p}=0.000)$. Coagulase-negative staphylococci were identified in $25.9 \%$ and $2.1 \%$ gram negative samples, in 4 cases was isolated Enterobacter sp. The alcohol-based handrub was effective when calculated before and handrubbing (RR 0.10 CI 0.02-0.39) when compared with the technique handwashing with soap and water (RR $0.12 \mathrm{CI} 0.03-0.5)$. The technique of handwashing was not effective (RR 0.71 CI 0.43-1.18)
}

Dra. Ángela Liliana Londoño F.: Especialista en Epidemiología. PhD en Medicina Preventiva y Salud Pública. Docente Facultad de Ciencias de la Salud, Universidad del Quindío; Lic. Marta Lucía Murillas B. Licenciada en Enfermería. Docente Facultad de Ciencias de la Salud, Universidad del Quindío. Armenia (Colombia)

Institución Financiadora: La investigación fue financiada por la Universidad del Quindío a través de proyecto aprobado en convocatoria interna.

Correspondencia. Ángela Liliana Londoño F. Armenia, Quindío (Colombia).

E-mail: angelalilianal@uniquindio.edu.co Recibido: 01/VI/2011 Aceptado: 27/X/2011 
Conclusion: Alcohol-based handrub prepared using the suggested recipe by the World Health Organization is more effective in removing germs than handwashing with soap. (Acta Med Colomb 2011; 36: 181-186)

Keywords: Handwashing, Cross Infection, hospital infections, Nosocomial Infections Infectious Disease Transmission, Professional-to-Patient, Infection Control, Communicable Disease Control, Health Care

\section{Introducción}

A principios del siglo XIX en Francia, se recomendó el uso de soluciones de cloruro como desinfectantes (1); posteriormente, el médico húngaro Ignaz Philipp Semmelweis, tras realizar amplias observaciones, asoció la "fiebre puerperal" a la existencia de una "materia cadavérica" que era transportada por las manos de médicos y estudiantes responsables de la atención de las mujeres en el periodo del parto, e implementó en un hospital de Viena en el año 1847 el uso de soluciones con cloro para limpieza de las manos antes y después de examinar o atender las pacientes, medida con la cual logró disminuir la mortalidad por dicha causa (1-3); de forma independiente el médico Oliver Wendell Holmes llegó a la misma conclusión (1); sin embargo, la recomendación del uso de las soluciones fue objeto de rechazo por gran parte de la comunidad médica de la época, aunque tuvo algunos seguidores y también fue implementada por Sir Joseph Lister como medida preventiva en las infecciones quirúrgicas $(2,4)$; sólo 40 años después Pasteur reconoció el mérito de Semmelweis al concluir que la fiebre puerperal era causada por "microbios transportados por los médicos y enfermeras de una paciente a otra" y demostró posteriormente que el Estreptococo era el agente causal (2).

Este descubrimiento ha sido de los más importantes en la historia de la medicina; poco a poco se fue aceptando la medida y en la actualidad la higiene de las manos se considera como la mejor estrategia y la más costo-efectiva en la prevención de las infecciones asociadas al cuidado de la salud (IACs) $(1,2,5)$.

Una buena higiene de las manos se puede conseguir utilizando un producto en base de alcohol o un jabón antibacteriano con un enjuague adecuado (6). Múltiples estudios (5,7-11) confirman la mayor eficacia de las soluciones alcohólicas que contienen etanol a $80 \%$ o alcohol isopropílico a 75\%; actualmente su utilización como alternativa al lavado de manos con jabón normal o con jabones antimicrobianos en la práctica hospitalaria, constituyen el objeto del Reto Mundial en pro de la Seguridad del Paciente "Una atención limpia es una atención más segura" (5). Esta investigación se realizó con el objeto de medir la eficacia de un preparado de base alcohólica para manos basado en la receta de la Organización Mundial de la Salud (OMS) (3) para la disminución de la flora bacteriana de las manos y al mismo tiempo compararla con el lavado de manos con agua y jabón.

\section{Material y métodos}

Se realizó un estudio cuasiexperimental en muestras relacionadas en una institución hospitalaria de la ciudad de
Armenia- Quindío; se invitaron a participar trabajadores sanitarios (TS) en los diferentes servicios de hospitalización y de urgencias; participaron voluntariamente y firmaron el consentimiento informado 103 TS. A pesar de tratarse de una muestra por conveniencia, se conservó la proporción de los participantes de acuerdo al total de médicos y personal de enfermería que laboraban en la institución. Se diseñó un instrumento con las variables de estudio.

\section{Hipótesis nula}

- El número de unidades formadoras de colonias (UFC) es igual antes y después de realizar lavado de manos con agua y jabón o de realizar higiene con solución alcohólica.

- Se diseñó un instrumento con las variables de estudio. Hipótesis de trabajo: el número de unidades formadoras de colonias (UFC) es menor después de realizar lavado de manos con agua y jabón o de realizar higiene con solución alcohólica.

\section{Variables de efecto}

Eficacia: disminución significativa de la carga bacteriana (en UFC) de las manos.

Protocolo de toma de muestras: las muestras fueron recolectadas por una enfermera investigadora con técnica estéril, tres horas después de iniciado el turno hospitalario y sin previo aviso. Para el lavado de manos se solicitó a los participantes utilizar el jabón que habitualmente utilizaban en los servicios donde estaban laborando y secarse como siempre lo hacían. Se explicó a cada participante como realizar la técnica de lavado o higiene de acuerdo con las directrices de la Organización Mundial de la Salud (OMS), que incluye los siguientes ítems: palmas, espacios interdigitales anterior y posteriores, dorso manos, dorso de dedos, pulgares y punta de los dedos; se escogió la mano dominante del participante y con hisopo se tomó muestra pasando por palma, espacios interdigitales y región periungueal (3). Se tomaron a cada participante, muestras para análisis microbiológico en cuatro momentos; momento 1) antes del lavado de manos con agua y jabón (LM), momento 2) a los cinco minutos del momento 1 después del LM. Pasado un mes, el momento 3) antes de la higiene con preparado de base alcohólica para manos que hemos llamado para el estudio "higiene con solución alcohólica" (HSA) y momento 4) a los cinco minutos se tomaba la segunda muestra después de la HSA.

Procesamiento de las muestras: se realizó en el Centro de investigaciones Biomédicas (CIBM) de la Universidad del Quindío. Para evitar la contaminación de ellas, se utilizó 
sólo equipo estéril y precauciones de asepsia; se tuvieron en cuenta las precauciones universales (bioseguridad). La solución alcohólica (SA) o preparado de base alcohólica para manos se preparó en el CIBM con técnica estéril y siguiendo las recomendaciones de la OMS (3) para obtener una concentración final de etanol a $80 \%$, glicerol $1.45 \%$, peróxido de hidrógeno $0.125 \%$; se mezclaron en un recipiente estéril graduado de $1000 \mathrm{ml}: 833.3 \mathrm{ml}$ etanol a $96 \%, 14.5 \mathrm{ml}$ glicerol al 98\%, y $41.7 \mathrm{ml}$ de peróxido de hidrogeno al 3\%. En el laboratorio se realizó conteo de unidades formadoras de colonias (UFC), con asa calibrada, cultivos en medios nutritivos (Agar Muller Hinton), coloración de Gram para la clasificación de los microorganismos, nuevo conteo de UFC y pruebas para identificación de Staphylococcus y Gram negativos.

Procesamiento y análisis de los datos: se realizó en el programa SPSS versión 14 y en el Epi-Info. Para medir la eficacia se calculó el riesgo relativo $(\mathrm{RR}=$ proporción de muestras positivas después del LM, HSA/proporción de muestras positivas antes del LM, HSA) y se midieron el riesgo atribuible (\% $\mathrm{RA}=$ proporción de muestras positivas antes del LM , HSA-proporción de muestras positivas después del LM, HSA/proporción de muestras positivas antes del LM, HSA x 100) y la fracción prevenible (\% Rap= proporción de muestras positivas en la población de estudio-proporción de muestras positivas después del LM, HSA/proporción de muestras positivas en la población de estudio x 100); el test de hipótesis para las variables cualitativas fue el de chi cuadrado y el nivel de significancia menor a 0.05 .

Aspectos bioéticos: se obtuvo aprobación del Comité de Bioética Institucional para la realización del estudio.

\section{Resultados}

Se realizó el estudio en un total de 103 trabajadores sanitarios (TS), $17.5 \%$ médicos (n:18) y $82.5 \%$ personal de enfermería (n: 85). Un $32.0 \%$ laboraban en UCI o quirófano, $26.2 \%$ en pediatría y recién nacidos, $17.5 \%$ en maternidad y sala de partos, $13.6 \%$ en medicina interna y $10.7 \%$ en servicios de hospitalización de pacientes quirúrgicos.
En la muestra previa al lavado de manos con agua y jabón (LM), tuvieron conteo positivo de unidades formadoras de colonias (UFC) un $28.3 \%$, mientras que en la muestra posterior al LM continuaron positivas un $15.3 \%$ de las muestras, con una disminución no significativa de las muestras positivas de $13 \%$ ( $p=0.19$ ) (Tabla 1$)$.

Al analizar los datos por parejas se encontró que entre los negativos en la primera muestra continuaron siendo negativos el $83.1 \%$ (n: 59) y se hicieron positivos el $16.9 \%$ (n: 12) en la muestra posterior al LM, entre estos últimos a $9(12.7 \%)$ se encontraron entre 1 y 15 UFC y a $3(4.2 \%)$ se identificaron 10000 o más UFC; así mismo, entre los positivos de la primera muestra se observó aumento de UFC en dos casos (el primero aumentó de 2 a 150 UFC y el segundo aumentó sólo de 1 a 6 UFC).

Con relación a la higiene con la solución alcohólica (HSA), se realizó en 82 participantes; 74.4\% (61 TS) tuvieron ausencia de UFC en la muestra previa y $97.6 \%$ (80 TS) en la muestra posterior a la higiene con SA. En un $25.6 \%$ se encontraron entre 0 y 15 UFC antes de la HSA y disminuyeron significativamente a 0 después de la SA. En dos casos que fueron negativos inicialmente se identificaron en la muestra después de la SA entre 1 y 15 UFC (Tabla 1).

\section{Etiología microbiana}

En las 332 muestras analizadas se identificaron Staphylococcus coagulasa negativo en el $25.9 \%$ (n: 64) y Gram negativos en $2.1 \%$ (n: 7).

Se identificaron Staphylococcus epidermidis antes del LM en 26 TS (92.9\% entre las muestras positivas y $26.3 \%$ entre todas las muestras tomadas), y posterior al LM en 16 TS $(80 \%$ entre las muestras positivas y $16.2 \%$ entre todas las muestras tomadas); antes de la HSA en 21 TS (100\% entre las muestras positivas y $20.4 \%$ entre todas las muestras tomadas), posterior a la HSA solo se identificó S. epidermidis sólo en una muestra.

Cocobacilos Gram negativos y Enterobacter sp se hallaron cada uno en un caso en $2 \mathrm{TS}$ antes del LM y aumentaron

Tabla 1. UFC* antes y después de cada técnica de higienización de manos.

\begin{tabular}{|c|c|c|c|c|c|c|c|c|}
\hline \multirow{3}{*}{ UFC } & \multicolumn{4}{|c|}{ Lavado manos } & \multicolumn{4}{|c|}{ Solución alcohólica } \\
\hline & \multicolumn{2}{|c|}{ Antes } & \multicolumn{2}{|c|}{ Después } & \multicolumn{2}{|c|}{ Antes } & \multicolumn{2}{|c|}{ Después } \\
\hline & No. & $\%$ & $\%$ & No. & No. & $\%$ & No. & $\%$ \\
\hline $1-15$ & 21 & 21.2 & 15 & 15.2 & 21 & 25.6 & 2 & 2.4 \\
\hline $16-99$ & 0 & 0 & 0 & 0 & 0 & 0 & 0 & 0 \\
\hline $100-999$ & 0 & 0 & 1 & 1,0 & 0 & 0 & 0 & 0 \\
\hline $1000-9999$ & 1 & 1.0 & 0 & 0 & 0 & 0 & 0 & 0 \\
\hline $10000 \mathrm{Y}+$ & 6 & 6.1 & 4 & 4.0 & 0 & 0 & 0 & 0 \\
\hline Subtotal positivos & 28 & 28.3 & 20 & 20.2 & 21 & 25.6 & 2 & 2.4 \\
\hline Negativos & 71 & 71.7 & 79 & 79.8 & 61 & 74.4 & 80 & 97.6 \\
\hline
\end{tabular}


después del LM a dos hallazgos de cada uno en 4 TS; antes de la HSA no se identificaron Gram negativos pero después de la técnica se identificó Enterobacter sp en 1 TS (Figura 1).

\section{Eficacia del LM y de la HSA}

No se encontraron diferencias entre las UFC halladas antes y después del LM $(p=0.18)$. Se encontraron diferencias significativas al comparar número de UFC antes y después de utilizar HSA $(p=0.000)$ y al comparar UFC con las dos técnicas (después del LM y de la HSA) $(p=0.000)$. La solución alcohólica resulto ser eficaz tanto cuando se calculó antes y después (RR 0.10 IC 95\% 0.02-0.39) como al compararla con la técnica de lavado con agua y jabón (RR 0.12 IC 95\% 0.03-0.5). En cambio la técnica de lavado de manos no resultó ser eficaz (RR 0.71 IC 95\% 0.43-1.18) (Tabla 2).

\section{Discusión}

Las infecciones relacionadas con la atención sanitaria (IRAS) se encuentran entre las principales causas de muerte y de incremento de la morbilidad en pacientes hospitalizados; aunque tienen etiología multicausal, la mayoría de ellas pueden evitarse con prácticas baratas y sencillas, entre las cuales la higiene de las manos es la medida primordial

Tabla 2. Eficacia del LM y de la HSA para eliminar los gérmenes de las manos

\begin{tabular}{|lccc|}
\hline Riesgo & $\begin{array}{c}\text { UFC después y } \\
\text { antes LM }\end{array}$ & $\begin{array}{c}\text { UFC después y } \\
\text { antes HSA }\end{array}$ & $\begin{array}{c}\text { UFC después de } \\
\text { HSA y de LM }\end{array}$ \\
\hline RR $(\text { IC } 95 \%)^{\mathrm{a}}$ & $0.71(0.43-1.18)$ & $0.1(0.02-039)$ & $0.12(0.03-0.5)$ \\
\hline $\mathrm{Chi}^{2} p$ & $1.76(0.18)$ & $16.4(0.000)$ & $11.6(0.000)$ \\
\hline$\% \mathrm{RA}^{\mathrm{b}}$ & 28.6 & 90.5 & 87.9 \\
\hline$\% \mathrm{RA} \mathrm{p}^{\mathrm{c}}$ & 16.7 & 85.6 & 80.0 \\
\hline $\begin{array}{l}\text { aRR: riesgo relativo; } \\
\text { cional; LM: Lavado } \text { Re manos; HSA: Higiene con solución alcohólica. }\end{array}$ \\
\hline
\end{tabular}

$(3,5,6)$. En la patogénesis de las IRAS la infección cruzada tiene un papel fundamental (5).

La flora bacteriana normal presente en la piel de las manos se clasifica como transitoria y residente, hacen parte de la flora normal Staphylococcus epidermidis, Staphylococcus aureus, Micrococcus, difteroides, especies no patógenas de Neisseria, Streptococcus del grupo A hemolíticos y no hemolíticos, especies de Propionibacterium y Peptoestreptococcus; también algunos Enterococcus y bacilos gram negativos coliformes $(3,12)$. La flora residente no es fácilmente modificada por el lavado mientras que la transitoria sí se puede eliminar con el lavado vigoroso o con desinfectantes; la flora transitoria puede estar presente durante horas o días y puede contener microorganismos tanto patógenos como no patógenos, es aquella que los trabajadores sanitarios adquieren durante el proceso de atención de pacientes, esta flora es superficial y de fácil eliminación con el lavado de manos y generalmente la causal en las IRAS, mientras que la flora residente es más profunda y los microorganismos que la componen son menos asociados a (IRAS) $(1,3,12)$.

Se ha calculado que la cantidad de bacterias en las manos de personal médico varía entre $3.9 \times 10^{4}$ y $4.6 \times 10^{6}$; en un estudio se informó que cuando los TS colocaban directamente la mano sobre una placa de agar, se contaban entre 0 y 300 UFC, siendo más propensos aquellos TS que tenían contacto directo y quienes manipulaban pacientes con afecciones respiratorias; también influía la duración de la actividad realizada $(1,3)$. Las manos de los TS se pueden contaminar con bacilos gram negativos, Staphylococcus aureus y otras bacterias hospitalarias; es común, la transmisión intrahospitalaria del virus sincitial respiratorio en unidades de cuidado infantil; de igual manera además de las manos también pueden contaminarse los guantes al tocar objetos en la vecindad del paciente $(1,3,5)$; en pacientes con MRSA se

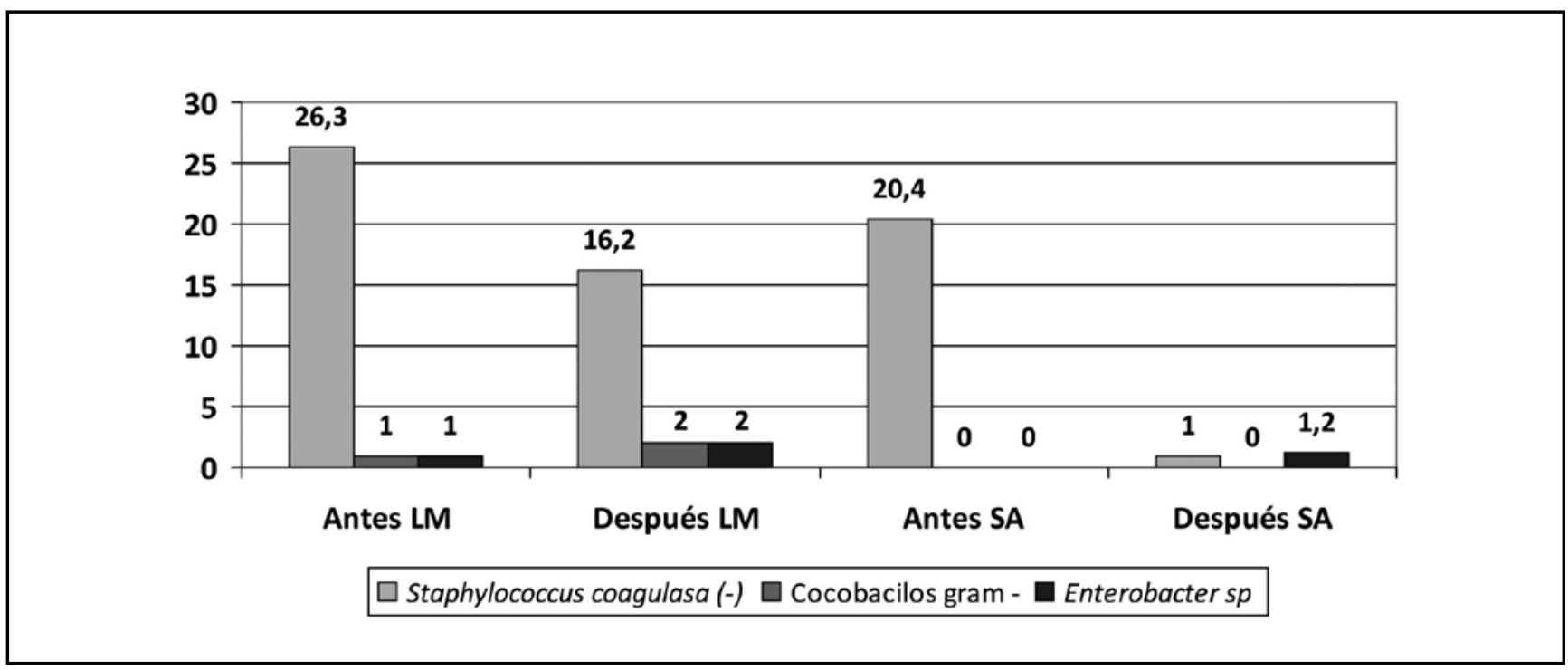

Figura 1. Gérmenes identificados en las manos de los trabajadores sanitarios antes y después de cada técnica de higiene de manos. LM=lavado de manos. 
ha demostrado que las superficies del cuarto se contaminan fácilmente (13).

La gran mayoría de gérmenes aislados en el presente estudio fueron cocos gram positivos tipo Staphylococcus coagulasa (-), los cuales se encontraron en las primeras muestras en la cuarta parte de los participantes. Los Staphylococcus tienen la característica de transmitirse de persona a persona o a través de fómites y pueden sobrevivir mucho tiempo en superficies secas (14); los Staphylococcus coagulasa (-) son actualmente los gérmenes que más frecuentemente se asocian a infecciones hospitalarias particularmente en unidades de cuidado intensivo neonatal donde se ha encontrado como germen etiológico hasta en el 78\% de las bacteriemias (15) siendo el Staphylococcus epidermidis el responsable de la mitad de estas infecciones con una elevada prevalencia de resistencia a antibióticos como la penicilina, la gentamicina y la oxacilina (15-19).

Los Gram negativos se encontraron en menor proporción, en cerca del 7\% de las muestras; llamó la atención el hallazgo de Enterobacter $s p$ en varias muestras pues es un germen que no hace parte de la flora normal de la piel y que suele ser uno de los gérmenes oportunistas asociados a infecciones hospitalarias en unidades de pacientes críticos con mayor frecuencia $(16,17,20,21)$. Enterobacter spp además del intestino humano, se puede encontrar en agua y alimentos; aunque ha sido aislado de equipos médicos, la mayoría de las IRAS provocadas por ellos son de origen endógeno en personas con enfermedades graves o debilitantes (22). Enterobacter spp se asocia a infecciones respiratorias, urinarias, bacteriemias, infecciones de herida quirúrgica, ortopédicas entre otras, con predominio en edades extremas. Presenta elevada resistencia, ya que produce betalactamasas de espectro extendido (BLEES) y su tratamiento es difícil porque además colonizan frecuentemente dispositivos intravasculares, sondas urinarias y ventiladores (21).

En el presente estudio, el lavado de manos con agua y jabón no demostró eficacia en la disminución de UFC en los cultivos, e incluso en algunos TS aparecieron después de realizar la técnica, probablemente porque el secado en su gran mayoría lo hacían con toalla de tela, ya que se pidió que lo hicieran de la forma que rutinariamente se realizaba en la institución. En cambio, la solución alcohólica preparada con la receta sugerida por la OMS demostró ser eficaz en la eliminación de gérmenes de las manos tanto cuando se comparó antes y después de su utilización, como cuando se comparó con la técnica de lavado con agua y jabón.

Las soluciones eficaces con base en alcohol contienen alcohol etílico o isopropílico en concentraciones que varían entre 60 y $90 \%(1,6,11)$. En cuanto a la eficacia de las soluciones alcohólicas (SA), numerosas publicaciones han demostrado que eliminan bacterias de las manos con más eficacia que el lavado con agua y jabón, y aun jabones antimicrobianos; así mismo, se ha demostrado el costo beneficio de las SA en la prevención de las IAC $(1,5,23)$. Los jabones normales tienen la capacidad de eliminar la suciedad pero carecen de efecto antimicrobiano; sin embargo, la duración del LM con jabones corrientes sí puede influir en la carga bacteriana; se ha establecido que el LM durante 15 segundos puede disminuir la flora transitoria en 0.6-1.1 $\log 10$ y durante 30 segundos la reduce 1.8-2.8 $\log 10$, pero también se ha encontrado que puede existir un aumento de carga bacteriana después del LM, ya que estos jabones pueden contaminarse; además tienen como desventaja que pueden provocar resequedad o irritación de la piel cuando las actividades asistenciales requieren que se laven las manos muchas veces (1); también para el LM se requieren toallas de papel, ya que tanto las de tela como el aire caliente pueden contaminar las manos (9). La actividad antimicrobiana del alcohol consiste en la desnaturalización de las proteínas, tiene elevada actividad germicida in vitro contra bacterias gram positivas y gram negativas incluyendo organismos multirresistentes, micobacterias, hongos y virus como el VIH, influenza y VSR; sin embargo, su eficacia es limitada contra esporas, protozoos, quistes, y algunos virus sin envoltura $(1,7,11)$. Frotar las manos durante 30 segundos con una solución que tenga alcohol a las concentraciones recomendadas puede disminuir la flora en $3.5 \log 10 \mathrm{y}$ si se hace durante un minuto en 4-5 $\log 10$, pero se debe tener en cuenta que su actividad residual es poca (1). Entre las ventajas de utilizar las soluciones alcohólicas, además de su acción desinfectante, se encuentra la reducción en la irritación de la piel de las manos cuando se usan con emolientes $(7,24)$ y la disminución del tiempo requerido para cumplir con las indicaciones de higiene de las manos durante la atención del paciente (23).

Una de las grandes dificultades a la hora de impactar sobre la incidencia de las IRAS con la medida de higiene de las manos, es la actitud de las personas en general que muchas veces no es modificada por el conocimiento como puede ocurrir con algunos TS; a través del tiempo se han probado estrategias educativas, de promoción e incluso de presión social por parte de los pacientes sin que se logren grandes modificaciones en esta conducta; sin embargo, la introducción de dispensadores con adecuada disponibilidad para el personal de salud, cerca a la cama de los pacientes, demuestra que los TS limpian sus manos significativamente más que cuando tienen que buscar un lavamanos disponible para el lavado de manos, por lo tanto esta medida aumenta la adherencia a la práctica de higiene de manos $(23,25)$.

Por último, vale la pena recordar la recomendación de la OMS sobre los cinco "momentos" o indicaciones para realizar higiene de manos con soluciones con base en alcohol: antes del contacto con el paciente, antes de realizar una tarea aséptica, después del riesgo de exposición a fluidos corporales, después del contacto con el paciente y después del contacto con el entorno o vecindad del paciente (5).

En conclusión, es necesario realizar educación continua sobre la utilización de soluciones alcohólicas para la higiene de manos y los momentos en que se indican, porque está demostrado que son más eficaces y costo efectivas; atender a las recomendaciones sobre el secado de manos con toallas de 
papel dada la posibilidad de contaminar las mismas cuando se hace con toallas de tela. Por último, se recomienda la preparación y utilización de la solución alcohólica en cada institución con las recetas de la OMS, porque además de más económicas garantizan el contenido de alcohol necesario para disminuir la carga bacteriana de las manos, lo cual no logran muchas soluciones comerciales, que además pueden ser muy costosas.

\section{Referencias}

1. Centers for Disease Control and Prevention. Guideline for Hand Hygiene in Health-Care Settings: Recommendations of the Healthcare Infection Control Practices Advisory Committee and the HICPAC/SHEA/APIC/IDSA Hand Hygiene Task Force. Centers for Disease Control and Prevention 2002; 51: 1-48

2. Miranda M, Navarrete L. Semmelweis y su aporte científico a la medicina: Un lavado de manos salva vidas. Rev Chil Infect 2008; 25: 54-7.

3. World Health Organization. WHO Guidelines on Hand Hygiene in Health Care First Global Patient Safety Challenge Clean Care is Safer Care. World Health Organization; 2009.

4. Casanova LJ, Castañón JA. Reflexiones acerca del lavado de manos. Rev Med; 2004; 42: 519-24.

5. Organización Mundial de la Salud. Guía de aplicación de la estrategia multimodal de la OMS para la mejora de la higiene de las manos. OMS 2009, GinebraSuiza

6. Arévalo JM, Arribas JL, Hernández MJ, et al. Guía de utilización de antisépticos. Sociedad española de Medicina preventiva; Salud pública e higiene 2001.

7. Daniel SE, De Mello AC, Sant'ana JJ, Sarto V, Cassiolato V, Botelho L, Cardoso CL. The effectiveness of alcohol gel and other hand-cleansing agents against important nosocomial pathogens. Braz J Microbiol 2004; 35: 33-9.

8. Ángeles U, Molinar F, Anaya VE, López ME. Efectividad de la aplicación de alcohol gel en la higiene de las manos de enfermeras y médicos. Rev Enferm IMSS 2005; 13: 15-21

9. Yamamoto Y, Ugai K, Takahashi Y. Efficiency of hand drying for removing bacteria from washed hands: comparison of paper towel drying with warm air drying. Infect Control Hosp Epidemiol 2005; 26: 316-20.

10. Pellowe CM, Pratt RJ, Loveday HP, Harper P, Robinson N, Jones S. The epic project. updating the evidence-base for national evidence-based guidelines for preventing healthcare-associated infections in NHS hospitals in England: a report with recommendations. J Infect Prev 2004; 5: 10-6.
11. Boyce JM. Using alcohol for hand antisepsis: dispelling old myths. Infection control and hospital epidemiology 2000, Ed. 21: 438- 41.

12. Brooks GF, Butel JS, Morse SA. Microbiología médica. México Editorial El Manual Moderno; 2005; Cap. 11: 193-8.

13. Boyce JM, Potter-Bynoe G, Chenevert C, King T. Environmental contamination due to methicillin-resistant Staphylococcus aureus: possible infection control implications. Infect Control Hosp Epidemiol 1997; 18: 622-7.

14. Murray PR, Rosenthal KS, Pfaller MA. Microbiología Médica. Madrid Elsevier España; 2007; Cap. 22: 221-36.

15. Álvarez M, Velazco E, Nieves B, Alviarez E, Araque M, Salazar E, Gutiérrez B. Caracterización fenotípica de cepas de Staphylococcus coagulasa negativo aisladas de una unidad de alto riesgo neonatal. Kasmera 2008; 36: 7-16.

16. Romero A; Martínez G, Flores O, Vásquez A. Perfil epidemiológico de las infecciones nosocomiales en un hospital de alta especialidad en el sureste mexicano. Salud en Tabasco 2007; 13: 618-24.

17. González N, Castañeda JL, Saltigeral P, Rodríguez MA, López C, Rosas A García E, et al. Infecciones nosocomiales en la Unidad de Cuidados Intensivos Neonatales del Instituto Nacional de Pediatría. Acta Pediatr Mex 2011;32: 28-32.

18. Craft AP, Finer NN, Barrington KJ. Vancomicina para la profilaxis contra la sepsis en recién nacidos prematuros (Revisión Cochrane traducida). Cochrane Plus 2006. número 2. Oxford, Update Software Ltd.

19. Galiana GG, Mangues MA, Demestre X, Farré R, Lopes AP, Altirriba $O$. Comportamiento farmacocinético de la vancomicina en neonatos de edad postconcepcional $\leq 32$ semanas, Propuesta de una pauta de dosificación. An Esp Ped 1996; 45: 167-71.

20. Salazar T, Morejón D, Alonso T, Ayala JI, López M, Castillo B. Gérmenes nosocomiales más frecuentes en la unidad de terapia intensiva. Rev Cub Med Int Emerg 2006; 5: 302-311.

21.Domínguez O, Hernández JC, Piedrahita N, Saldarriaga N, Vanegas DM, González JC. Características clínico-epidemiológicas de las infecciones por Enterobacter en la Clínica Cardiovascular de Medellín, agosto de 2004 a agosto de 2006. MEDICINA UPB 2008; 27: 101-11.

22. Cobo FJ, Rodríguez M. Infecciones por enterobacterias oportunistas. Tratado SEIMC de Enfermedades infecciosas y Microbiología Clínica. Buenos Aires, Madrid; Médica Panamericana 2005. Cap. 29: 337-45

23. Boyce JM. Antiseptic technology: access, affordability and acceptance. Emerg Infect Diseases 2001; 160: 231-3.

24. Boyce JM, Kelliher S, Vallande N. Skin irritation and dryness associated with two hand hygiene regimens: soap and water handwashing versus hand antisepsis with an alcoholic hand gel. Infect Control Hosp Epidemiol 2000; 21: 442-8.

25. Bischoff WE, Reynolds TM, Sessler CN, Edmond MB, Wenzel RP. Handwashing compliance by health care workers. The impact of introducing an accessible, alcohol-based hand antiseptic. Arch Intern Med 2000; 160: 1017-21. 